\title{
A PIV COMPARISON OF THE FLOW FIELD AND WALL SHEAR STRESS IN RIGID AND COMPLIANT MODELS OF HEALTHY CAROTID ARTERIES
}

\author{
PATRICK H. GEOGHEGAN \\ Department of Mechanical Engineering, University of Canterbury, Private Bag 4800, \\ Christchurch 8041, New Zealand \\ patrick.geoghegan@canterbury.ac.nz \\ MARK C. JERMY \\ Department of Mechanical Engineering, University of Canterbury, Private Bag 4800, \\ Christchurch 8041, New Zealand \\ mark.jermy@canterbury.ac.nz \\ David S. Nobes \\ Department of Mechanical Engineering, University of Alberta, \\ 4-31B Mechanical Engineering Building, Edmonton, AB, T6G 2G8, Canada \\ david.nobes@ualberta.ca
}

\begin{abstract}
Certain systems relevant to circulatory disease have walls which are neither rigid nor static, for example, the coronary arteries, the carotid artery and the heart chambers. In-vitro modelling allows the fluid mechanics of the circulatory system to be studied without the ethical and safety issues associated with animal and human experiments. Computational methods, in which the equations are coupled governing the flow and the elastic walls are maturing. Currently there is a lack of experimental data in compliant arterial systems to validate the numerical predictions. Previous experimental work has commonly used rigid wall boundaries, ignoring the effect of wall compliance. Particle Image Velocimetry is used to provide a direct comparison of both the flow field and wall shear stress observed in experimental phantoms of rigid and compliant geometries representing an idealized common carotid artery. The input flow waveform and the mechanical response of the phantom are physiologically realistic. The results show that compliance affects the velocity profile within the artery. A rigid boundary causes severe over estimation of the peak wall shear stress with a maximum relative difference of $61 \%$ occurring; showing compliance protects the artery from exposure to high magnitude wall shear stress. This is important when trying to understand the development of diseases like atherosclerosis. The maximum, minimum and time averaged wall shear stress in the rigid geometry was 2.3, 0.51 and $1.03 \mathrm{~Pa}$ and in the compliant geometry $1.4,0.58$ and $0.84 \mathrm{~Pa}$ respectively.
\end{abstract}

Keywords: PIV, wall shear stress, carotid artery, compliant

\section{Introduction}

Several common diseases involve the flow of a biofluid in a compliant (meaning it is distensible and undergoes elastic deformation when a force is applied) vessel. Atherosclerosis is caused by the formation of atherosclerotic plaque, which causes a progressive constriction of the arterial wall. Atherosclerosis is most serious when it affects the carotid or coronary arteries. In the carotid artery, it can lead to stroke, with 795000 people experiencing a new or recurrent stroke and 130000 fatalities in the U.S.A per year. ${ }^{1}$ In the coronary artery it causes coronary heart disease, which causes 400000 fatalities a year in the USA. ${ }^{1}$ It is expected that arterial wall movement has a significant effect on the flow field developed within arterial structures, affecting the wall shear stress (WSS), which plays an important part in the development of atherosclerosis.

WSS is a major influence on the structure of the endothelium..$^{2,3}$ In the main vasculature, in regions void of arterial geometry change, the arterial vessel diameter changes to maintain a normal physiological WSS $\sim 1-2 \mathrm{~Pa}^{2}$ A long-term increase in WSS experienced in an artery results in vasodilation followed by a remodeling of the artery to a larger diameter with the same arterial structure. A low WSS causes a thickening of the intimal layer (producing a stenosis) to re-establish a normal WSS. ${ }^{4}$ 
There are several published reviews of techniques that can be applied to numerical and experimental studies of the arterial system. ${ }^{4-7}$ It is currently very difficult to investigate in-vivo, the interaction between blood flow and the vessel wall. Vennemann et al. ${ }^{8}$ discuss the possibility of the use of Laser Doppler Velocimetry (LDV), but there is a requirement of optical access to the area of interest, which would require invasive surgery to provide this. Vennemann et al. ${ }^{8}$ also discuss the use of a non-invasive technique known as ultrasound particle image velocimetry, but the spatial resolution is limited. Elkins et al. ${ }^{9}$ provide an in depth review of Magnetic Resonance Velocimetry which utilizes Magnetic Resonance Imaging (MRI). Currently the resolution of in-vivo methods is limited, though great improvements are anticipated. Ultrasound and echocardiography can be used to obtain in-plane velocity components, but require assumptions to be made of the velocity profiles for flow quantification. ${ }^{5}$

In-vitro modelling with artificial flow phantoms allows the fluid mechanics of the circulatory system to be studied without the ethical and safety issues associated with animal and human experiments. Extensive work has been performed using both experimental and computational techniques to study rigid models ${ }^{10-14}$ representing the arterial system. Computational methods, in which equations are coupled governing the flow and the elastic walls are maturing. ${ }^{15-18}$ There is a lack of experimental data in compliant arterial systems to validate the numerical predictions with only a few publications available. ${ }^{19-21}$

Early work with healthy arterial geometries by Perktold et al. ${ }^{22}$ numerically investigated the effect of compliance on a geometry representing the human common carotid artery (CCA) bifurcation experiencing a physiologically realistic waveform making observations of both the WSS and vessel compliance. There remained a need for further development to make the wall mechanics far more physiologically realistic with more attention paid to boundary conditions. Yamaguchi et al. ${ }^{6}$, Gerbeau et al. ${ }^{23}$, Tezduyar et al. ${ }^{24}$, Tezduyar et al. ${ }^{25}$, Takizawa et al. ${ }^{26}$ present methods for numerically modelling fluid structure interaction (FSI) in biofluid problems with test case flow field results and WSS data. The very fine mesh required to obtain accurate WSS results is a restricting factor in this work. Recently Moireau et al. ${ }^{16}$ has presented a method for the identification of boundary support parameters in a FSI model using patient specific data with the aim that in future work it is applied in complete patient specific vascular modelling.

Even though FSI codes are maturing, it can be seen that there is still a long way to go till they can be readily applied to a cardiovascular problem. They still require experimental validation especially when understanding the boundary conditions that should be applied to the geometries to obtain the correct structural response to the pressure development experienced as this has not been fully clarified in work to date.

Experimental analysis of the FSI experienced by an elastic flow phantom using optical techniques has been previously reported for certain flow regimes. LDV has been used to study the phenomenon of flow rate limitation ${ }^{27,28}$ found most commonly in the systemic veins. $\mathrm{Ku}$ et al. ${ }^{29}$ applied LDV to the study of pulsatile flow in a rigid model of the carotid artery bifurcation Eguchi et al. ${ }^{30}$ investigated the applicability of using PIV to study flow in an elastic vessel experiencing a physiological flow wave in a generic peripheral artery. This waveform varied markedly from the CCA waveform studied here as it incorporated a small region of reverse flow. The results were compared to that of a rigid phantom. It was found that estimated flow rate obtained via integration of a 2-D PIV flow field between rigid and compliant phantom varied, especially in regions of maximum growth and shrink rate of the phantom radius. Pielhop et al. ${ }^{21}$ investigated the effect of an idealized sinusoidal flow input on an elastic vessel and the WSS experienced. There have been extensive experimental investigations into rigid geometries representing 
the carotid artery, with in depth analysis of the flow field and WSS encountered. There however seems to be very little experimental investigation into the effect compliance has on this ${ }^{21,31-33}$ especially with respect to a physiologically realistic flow input. A direct comparison needs to be made between a compliant and rigid geometry experimentally to validate numerical work and to ascertain the role compliance plays in the arterial system.

\section{Experimental Methodology}

The structure of the CCA varies widely throughout the general population. ${ }^{34}$ Idealized geometries can be used to study the general flow field and WSS experienced and help develop an understanding of the results expected. The construction process becomes inherently more complex when the flow phantom wall is compliant. Using the assumption of an idealized geometry alleviates further increase in complexity. This work is also in part, meant as a source of validation for current and future computational

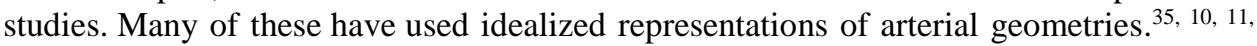

13 That being said, it is acknowledged that geometric variation from person to person will have an influence on hemodynamic factors, especially when the geometry changes from a healthy artery, to an artery with atherosclerosis. ${ }^{20,36}$

The compliant (Fig. 1) and rigid (Fig. 2) flow phantoms were produced by the method described in Geoghegan et al. ${ }^{37}$ from silicone (Dow Corning Sylgard 184). They were constructed at a scale of 3.2 times life size to increase the spatial resolution in the velocity and WSS measurements. The internal diameter of the rigid phantom matched the unstressed neutral diameter of the compliant phantom $(20 \mathrm{~mm})$.

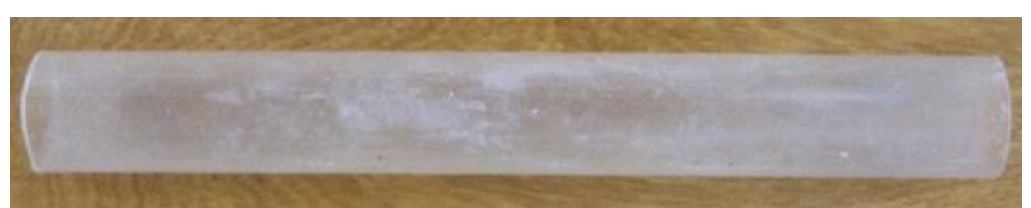

Fig. 1 Thin walled phantom representing a healthy CCA.

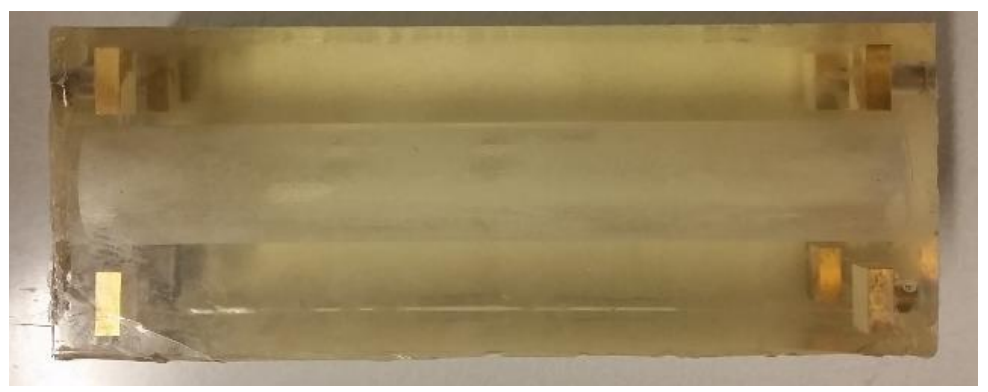

Fig. 2 Rigid walled phantom representing a healthy CCA.

To ensure a physiologically realistic mechanical response in compliant phantom the distensibility (also known as compliance) has to be matched to that of the CCA using Eq. $(1)^{32,38}$ where $\mathrm{d}$ is the distensibility, $\mathrm{A}$ is the cross-sectional area of the cylindrical vessel, $\mathrm{p}$ is the pressure, $\mathrm{E}$ is the Young's Modulus, $\mathrm{h}$ is the wall thickness and $\mathrm{D}$ is the unstressed neutral diameter. Table 1 provides an overview of both the properties of the $\mathrm{CCA}^{39}$ and the compliant flow phantom. Using Eq. 1 and the data from Table 1 gives the CCA a distensibility of $1.29 \times 10^{-5} \mathrm{~m}^{2} / \mathrm{N}$. It should be noted that Riley et al. ${ }^{39}$ show the Young's Modulus of the CCA varies between $771 \mathrm{kPa}$ and $983 \mathrm{kPa}$ and an average of 877 
$\mathrm{kPa}$ was used for this work. With the Young's Modulus of the silicone and the unstressed diameter of the phantom known, the phantom required a wall thickness of $1.32 \mathrm{~mm}$ to ensure a match in distensibility. The compliant phantom had a wall thickness of $1.28 \pm 0.05 \mathrm{~mm}$, which accounting for experimental error, produced a physiologically realistic response.

$$
d=\frac{1}{E(h / D)} .
$$

Table 1. $\mathrm{CCA}^{39}$ and flow phantom properties.

\begin{tabular}{cccc}
\hline Property & CCA & $\begin{array}{c}\text { Healthy Rigid } \\
\text { Flow Phantom }\end{array}$ & $\begin{array}{c}\text { Healthy Compliant Flow } \\
\text { Phantom }\end{array}$ \\
\hline Wall Thickness $(\mathrm{mm})$ & 0.617 & N/A & 1.28 \\
Internal Diameter $(\mathrm{mm})$ & 6.2 & 20 & 20 \\
Density(blood, glycerol) $\left(\mathrm{kg} / \mathrm{m}^{3}\right)$ & 1060 & 1150 & 1150 \\
Young's Modulus $(\mathrm{kPa})$ & $771-983$ & N/A & 1320000 \\
Time Period Oscillation $(\mathrm{s})$ & 1 & 2.92 & 2.92 \\
Length $(\mathrm{mm})$ & 100 & 210 & 210 \\
\hline
\end{tabular}

A schematic of the experimental flow system is shown in Fig. 3. This setup had the phantom orientated in the vertical direction. Steady flow was provided by a header tank with weir (1). The working fluid passed through a flow straightener in a settling chamber (5), attached to $1.5 \mathrm{~m}$ long pipe, which reduced flow vorticity providing stable and fully developed flow at the phantom inlet. An electromagnetic flow meter (Krohne Optiflux 1300) was attached just upstream of the phantom to provide real-time entrance flow rate data. The phantom was encased in an external pressurization chamber (2), which served 2 purposes. Firstly, it contained the same water/glycerine mixture as the working fluid to minimize diffraction caused by the curvature of the phantom. Secondly, it ensured there was a constant static pressure across the phantom wall. Temperature stability was controlled by a feedback temperature control system. Experimental analysis was performed at the longitudinal center of the compliant and rigid phantom (Fig. 4).

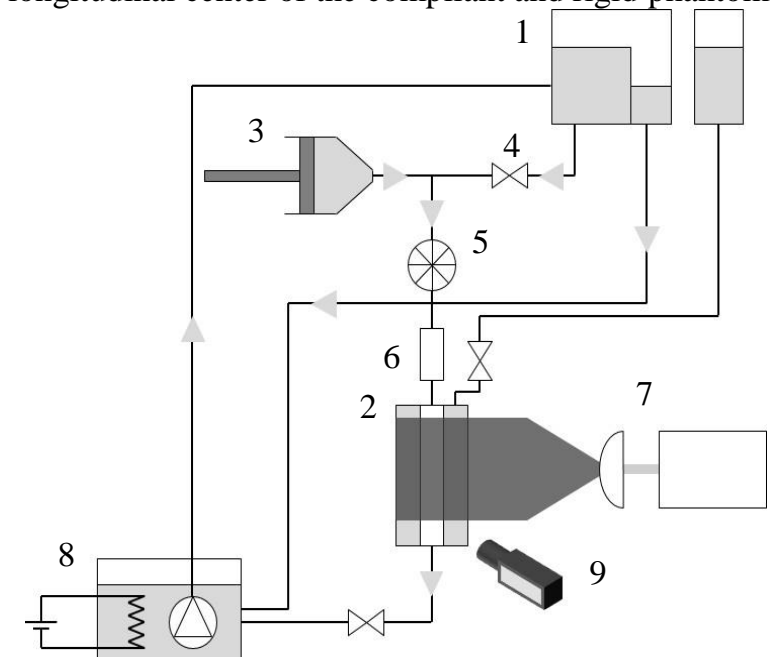

Fig. 3 Schematic view of flow system setup, the arrows represent flow direction (1) header tanks with weir for constant pressure (2) external pressure chamber (3) Compressed air piston pump (4) ball valves for flow control (5) flow straightener (6) electromagnetic flow meter (7) laser and optics (8) sump with pump and cooling unit (9) PIV camera. 


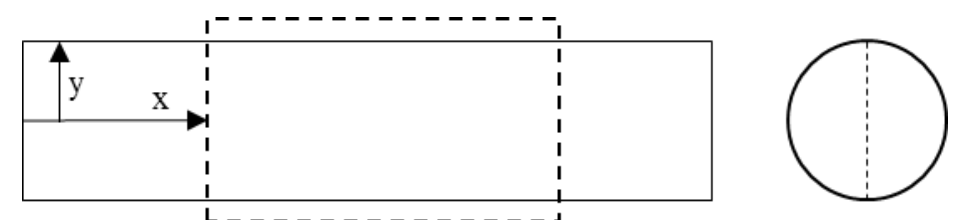

Fig. 4 (Left) Schematic showing the region of interest (dotted region) recorded through PIV experimentation. All analysis was performed at the central longitudinal location. The coordinate system is also provided for reference. (Right) Cross section of the phantom with the dotted line representing the plane used for experimental analysis

To ensure dynamic similarity so that the experimental conditions of the 3.2 scaled invitro phantom matched in-vivo conditions, firstly the Reynolds number was matched $(R e)$ (Eq. (2)). Secondly, to ensure similarity in oscillatory flow, the Womersley number ${ }^{40}(\alpha)$ (Eq. (3)) was matched. The Womersley number is a combination of the Strouhal number and the Re and is commonly used in biological fluid flow problems. $U$ is the mean inlet velocity, $\omega$ is the frequency of pulsation and $v$ is the kinematic viscosity. The resulting invitro inlet waveform for the compliant and rigid phantom is displayed in Fig. 5 along with the in-vivo waveforms in the CCA of a healthy male volunteer. ${ }^{41}$ The compliant and rigid waveforms match closely during the initial acceleration and deceleration phase, as flow rate plateaus the compliant waveform has a higher flow rate with the waveforms converging in late diastole. Overall the match is satisfactory. The in-vitro waveform had a maximum, minimum and mean Re of 953, 423 and 614 respectively and an $\alpha$ of 4.54, closely matching in-vivo conditions. The working fluid was a mixture of $39 \%$ water and $61 \%$ glycerine by weight with a refractive index that matched the silicone phantom $(n=$ $\left.1.415^{37}\right)$. It had a kinematic viscosity $(v)$ of $10.2 \times 10^{-6} \mathrm{~m}^{2} / \mathrm{s}$ and a density $(\rho)$ of 1150 $\mathrm{kg} / \mathrm{m}^{3}\left(1.15 \mathrm{~g} / \mathrm{cm}^{3}\right)$ at $20{ }^{\circ} \mathrm{C}$. Blood is considered a non-Newtonian fluid; its viscosity varies with shear rate. The non-Newtonian behavior of blood is highly dependent on shear rate and hematocrit level ${ }^{42}$. The non-Newtonian behavior of blood is neglected in this study. This assumption is common place in experimental and numerical modelling of arterial fluid dynamics in medium to large arteries. In these geometries the shear rates are regularly above $100 \mathrm{~s}^{-1}$ which is large enough that the groups of red blood cells have separated so much that they no longer affect the blood viscosity ${ }^{43}$.

$$
\begin{aligned}
& R e=\frac{U D}{v} . \\
& \alpha=D \sqrt{\frac{\omega}{v}} .
\end{aligned}
$$




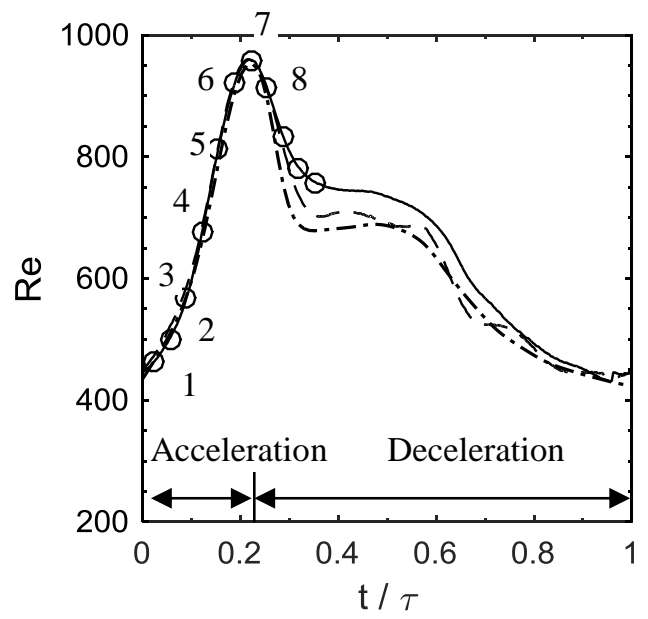

Fig. 5 Pulsatile in-vivo (•-), in-vitro rigid phantom (--) and in-vitro compliant phantom (-) inlet waveform with discrete points of interest numbered for convenience.

Time varying displacement fields in both the rigid and compliant phantom were recorded using planar digital PIV, which provided flow velocities and WSS data. Table 2 provides an overview of the measurement parameters. A single Dantec Flowsense 2MP camera with a Nikon $60 \mathrm{~mm}$ lens recorded the PIV images. The experiment had an estimated depth of field of $2 \mathrm{~mm}$ and diffraction limited particle size of $13 \mu \mathrm{m}$. A New Wave Solo $120 \mathrm{XT}$ laser with a wavelength of $532 \mathrm{~nm}$ at $120 \mathrm{~mJ} /$ pulse was passed through two convex lenses in a Keplerian arrangement to reduce the laser beam from a thickness of $5 \mathrm{~mm}$ to $0.5 \mathrm{~mm}$. A plano-concave lens is then used to diverge the beam into a laser light sheet which was $40 \mathrm{~mm}$ high and $0.5 \mathrm{~mm}$ thick. The flow was seeded with nominally 10 $\mu \mathrm{m}$ diameter hollow glass spheres which at $1.1 \mathrm{~g} / \mathrm{cm}^{3}$ were near neutrally buoyant in the water/glycerol mixture. Particle response time $\left(\tau_{\mathrm{s}}\right)$ to the fluid movement was $\sim 0.5 \mu \mathrm{s}$. 
Table 2 Table of measurement parameters, $\mathrm{C}$ and $\mathrm{R}$ refer to rigid and compliant respectively.

\begin{tabular}{lll}
\hline Laser & Type & Nd:YAG, dual cavity \\
& Thickness $(\mathrm{mm})$ & 0.5 \\
\hline Camera & Type & CCD \\
& Resolution (pix) & $1600 \times 1200$ \\
& Lens Focal Length $(\mathrm{mm})$ & 60 \\
& Lens Aperture (f\#) & 8 \\
& Magnification & 0.25 \\
\hline Seeding & Type & Hollow Glass Spheres \\
& Mean Diameter $(\mu \mathrm{m})$ & 10 \\
& Density $\left(\mathrm{g} / \mathrm{cm}^{3}\right)$ & 1.1 \\
\hline PIV & Window Sizing (pix) Max (Min) & $128 \times 128(32 \times 32)$ \\
& Resolution (pix/mm) & $38.75(\mathrm{C}) 49.95(\mathrm{R})$ \\
& Overlap $(\%)$ & 75 \\
& Vector Spacing $(\mathrm{mm})$ & $0.2(\mathrm{C}) 0.16(\mathrm{R})$ \\
& Time delay $(\mu \mathrm{s})$ & 1200 \\
\hline iPIV & Interrogation width, M (pix) & $32(\mathrm{C}) 64(\mathrm{R})$ \\
& Interrogation height, N (pix) & 80 \\
& Gaussian weight, $\sigma(\mathrm{pix})$ & 10 \\
& Overlap $(\%)$ & 75 \\
& Vector Spacing (mm) & $0.2(\mathrm{C}) 0.32(\mathrm{R})$ \\
\hline
\end{tabular}

The PIV measurements were phase locked, recording 30 image pairs per waveform over 15 waveforms. Spence et $\mathrm{al}^{44}$ showed that for ensemble averaged data there was a convergence of $0.03 \%$ between pairs 9 and 10 . In a time oscillating flow the laser pulse time delay selection has to be a compromise between the variation in pixel displacement for maximum and minimum flowrate. $1200 \mu \mathrm{s}$ was selected, which produced $<3 \%$ invalid vectors at each phase. After minimum background subtraction, Gaussian smoothing $(3 \times 3)$, intensity normalization and masking ${ }^{45}$, the images were processed with a 2D fast Fourier transform cross-correlation, with iterative window sizing (starting at $128 \times 128$ and reducing to $32 \times 32$ after 2 iterations) and displacement. ${ }^{46}$ The data was validated using the signal-to-noise ratio filter and normalized median test. ${ }^{47}$ Phase averaged results of the pulsatile flow are presented.

WSS was calculated using the interfacial PIV (iPIV) technique developed by Buchmann et al. ${ }^{48}$. Assuming that the flow in the boundary layer travels virtually parallel to the wall interface, the transformed images are discretized into interrogation windows $\mathrm{M} \times \mathrm{N}$ $\left(32 \times 80 \mathrm{pixel}^{2}\right)$ in size and a 1-D cross-correlation is performed on each horizontal line of the interrogation window. Strong tracer signals produce peaks and are located at a position representing the tracer displacement. The results produce a correlation peak at each horizontal with the location of the peaks lying along the velocity profile. The masked images produced for PIV experimentation provide the location of the wall interface required for WSS analysis. Following this a wall analysis region in the flow domain with constant height (80 pixels) in the wall normal direction is defined in physical $(\mathrm{x}, \mathrm{y})$ coordinates in the PIV images and can be described with a piecewise spline curve. Within the region, an orthogonal curvilinear grid is generated and mapped to a rectangular domain using conformal transformation to preserve orthogonality. The particle images are interpolated onto the rectangular grid with subpixel accuracy using a two-dimensional cardinal interpolation function. As previously stated, assuming the flow within this region moves predominantly parallel to the wall, a 1D correlation function between the two images can be calculated at every horizontal position within a region of 
interest. Previous error analysis of the iPIV technique has been performed by Buchmann et al. ${ }^{49}$. It was found that with a wall location accurate to \pm 1 pixel the iPIV produced an error in wall shear rate (WSR) of $\pm 0.02 \mathrm{pix} /$ pix equating to an error in WSS of $\pm 0.15 \mathrm{~Pa}$ ( $10 \%$ and $6 \%$ of the maximum WSS in the compliant and rigid phantom respectively). The technique was developed for a rigid wall system, but is still applicable in a moving wall system as there is negligible movement of the wall between images in a pair.

To assess the accuracy of the PIV analysis and the dimensionless scaling applied in this study, Eq. (4) was used to calculate the peak flow rate observed in both the compliant and rigid phantom PIV flow fields. The peak flow rate observed at the inlet from the electromagnetic flow meter on both the compliant and rigid phantom was $\operatorname{Re} 953$. Applying Eq. (4) to the velocity profiles in the compliant and rigid phantom produced a Re of 956 and 950 respectively; in both cases this produces a relative error of $0.03 \%$. The electromagnetic flow meter had a relative uncertainty of $1.3 \%$, combining this with an error of $1.9 \%$ due to temperature variation in the working fluid $\left(20 \pm 0.5{ }^{\circ} \mathrm{C}\right)$ provided an error in $\operatorname{Re}$ of $\pm 2.3 \%$.

$$
Q=\int u(r) 2 \pi r d r
$$

It is commonly expected that in PIV there is an error of 0.1 pixels in the calculated displacement. ${ }^{45}$ For the time delay and resolution given in Table 2, this equates to an error of $0.0021 \mathrm{~m} / \mathrm{s}\left(0.29 \%\right.$ of the maximum velocity $\left.\left(\mathrm{U}_{\max , \mathrm{c}}\right)\right)$ in the compliant phantom and $0.0016 \mathrm{~m} / \mathrm{s}\left(0.21 \%\right.$ of the maximum velocity in the rigid phantom $\left.\left(U_{\max , \mathrm{r}}\right)\right)$ in the rigid phantom. A misalignment of the light sheet by half the light sheet thickness $(0.25$ $\mathrm{mm}$ ) from the plane of symmetry would, for a fully 3D symmetrical flow, reduce the centerline velocity by $1 \%$.

WSS $\left(\tau_{w}\right)$ is calculated using Eq. (5). To provide physiological relevance all velocities and WSS data in this study are scaled to in-vivo conditions through Reynolds scaling using Eq. (6) and (7) respectively, with $\mu$ representing the dynamic viscosity, $\rho$ the density, and 3.2 is the scale factor between in-vitro and in-vivo phantom diameter. Data on the time averaged wall shear stress (TAWSS) is also presented, which over the course of one cardiac cycle is calculated by Eq. (8).

$$
\begin{gathered}
\tau_{w}=\mu \frac{\partial u}{\partial y} . \\
U(t)_{\text {in-vivo }}=3.2 \frac{v_{\text {in-vivo }}}{v_{\text {in-vitro }}} U(t)_{\text {in-vitro }} . \\
\tau_{w, \text { in-vivo }}=\frac{\rho_{\text {in-vivo }}}{\rho_{\text {in-vitro }}}\left(\frac{\mu_{\text {in-vivo }}}{\mu_{\text {in-vitro }}} 3.2\right)^{2} \tau_{w, \text { in-vitro }} . \\
T A W S S=\frac{1}{\tau} \int_{0}^{\tau} \tau_{w} d \tau .
\end{gathered}
$$

\section{Results and Discussion}

A comparison of compliant and rigid phase averaged streamwise velocity $\left(\left\langle U_{x}\right\rangle\right)$ normalized by the maximum velocity in the rigid phantom $\left(U_{\max , r}\right)$ over 1 periodic cycle is shown in Fig. 6, with the profile taken in the center of the phantom longitudinally. The results show the phase locations covering the acceleration phase, peak flow rate and the initial deceleration phase (Fig. 5 1-7). At peak flow rate the difference between inlet flow rates for the two phantoms was minimal $( \pm 3 \mathrm{Re})$ and the flow rate gradient leading up to the peak flow rate for both phantoms was also equivalent. The normalized phase 
averaged expansion and contraction of the compliant phantom is shown in Fig. 7 with wall position located using the wall detection technique described in Geoghegan et al. ${ }^{31}$. Through the acceleration phase the phantom diameter increased, in turn increasing the cross sectional area, which increased the volumetric capacity of the region of interest. At peak flow rate the phantom diameter increased to $1.13 \mathrm{D}$ (matching well to the $1.12 \mathrm{D}$ observed in-vivo by Sui et al. $^{50}$ ). For an equal flow rate input, an increase in cross sectional area reduces the velocity magnitude across the cross section. Along the center line the compliant phantom reached a peak velocity of $0.94 U_{\max , r}$. This reduces the gradient of the velocity profile at the wall when compared to the rigid phantom. This reduction in gradient occurs between $0 \leq t / \tau \leq 0.6$. When $t / \tau>0.6$ the gradient of the velocity profile has minimal difference between compliant and rigid phantoms.
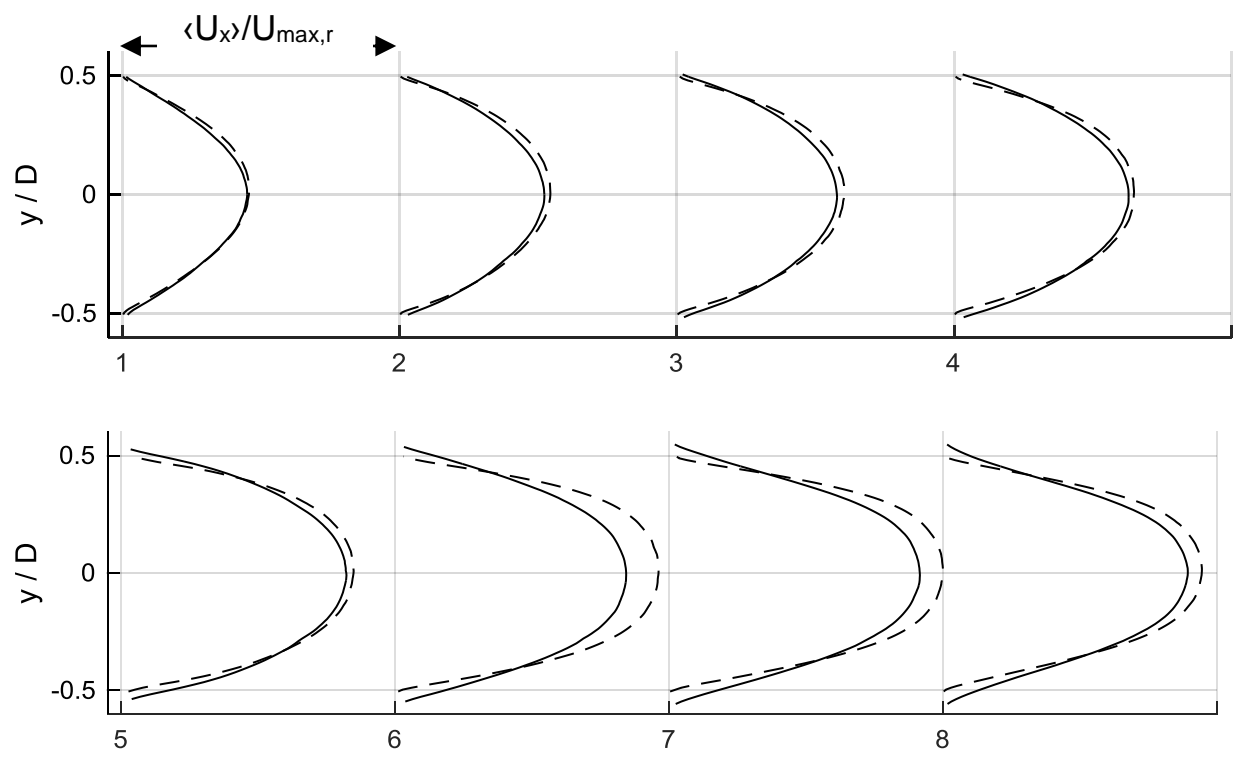

Fig. 6 Comparison of normalized phase averaged streamwise velocity profiles ( $\langle\mathrm{Ux}\rangle / \mathrm{Umax}, \mathrm{r})$ for both compliant (-) and rigid (--) geometry at selected phase locations indicated in Fig. 5.

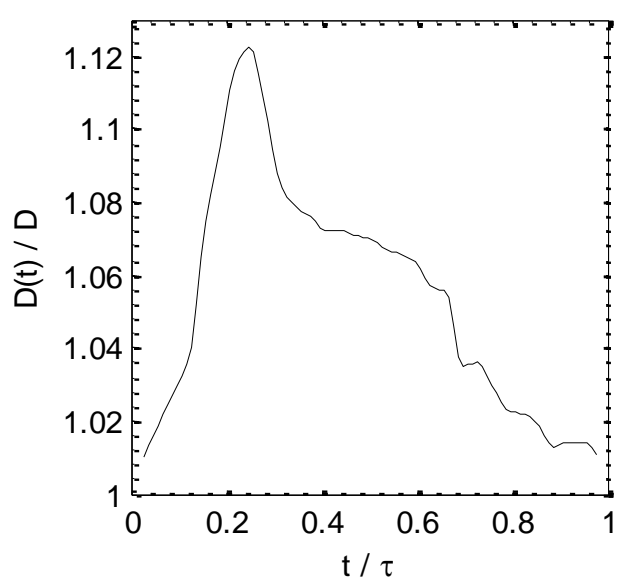

Fig. 7 Normalized phase averaged expansion and contraction of the compliant phantom at $\mathrm{x} / \mathrm{D}=0.25$. 
A comparison of the temporal evolution of the WSS observed in the compliant and rigid phantom is presented in Fig. 8. Results are presented for one wall only as there was minimal difference between the results obtained on opposite surfaces. The maximum, minimum and TAWSS in the rigid geometry was 2.3, 0.51 and $1.03 \mathrm{~Pa}$ respectively, and in the compliant geometry $1.4,0.58$ and $0.84 \mathrm{~Pa}$ respectively. A $40 \%$ reduction in peak WSS would in medical terms help avoid cell erosion ${ }^{51}$ and a $14 \%$ increase in minimum WSS would help keep the endothelial cells aligned ${ }^{2}$ and mechanically stimulated. There is also a $19 \%$ reduction in TAWSS. In the rigid phantom it is evident that there was a strong temporal variation in the WSS (Fig. 8), followed the inlet flow rate waveform path closely (Fig. 5). A sharp rise can be seen in the acceleration phase with a decrease during the deceleration phase. There is a marked difference between compliant and rigid WSS in this region $(0 \leq t / \tau \leq 0.4)$, with a maximum relative difference $(61 \%)$ occurring at peak flow rate $(\mathrm{t} / \tau=0.3)$. Eq. (5) shows WSS to be directly proportional to velocity gradient. Therefore, the reduction in gradient observed in Fig. 6 reduces the WSS. It shows an over prediction of WSS when the rigid-wall assumption is used. Further, it can be seen that at peak flow rate, the WSS actually reduces to its minimum value over the entire cardiac cycle. This is not observed in the rigid results, showing compliance actually ameliorates the WSS magnitude.

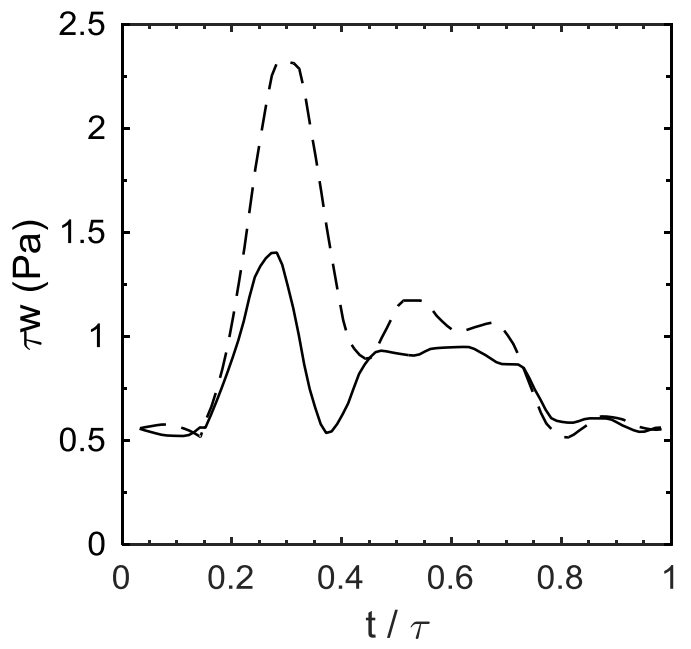

Fig. 8. Temporal evolution of the WSS in the compliant (-) and rigid phantom (--) at $\mathrm{x} / \mathrm{D}=0.25$.

To understand the cause of the variation in WSS between the compliant and rigid phantom a comparison of the normalization (achieved using Eq. (9) where $F(t)$ is the time varying component $(\mathrm{D}(\mathrm{t}), \mathrm{WSS}(\mathrm{t})$ or $\mathrm{U}(\mathrm{t}))$ and $\bar{F}(t)$ is the average value of $\mathrm{F}(\mathrm{t}))$ of the centerline velocity, expansion and contraction and WSS is performed in the compliant phantom (Fig. 9(1)). The same comparison between the centerline velocity and WSS in the rigid phantom is also presented (Fig. 9(2)). In the rigid phantom a phase shift between the waveforms was observed, with the peak WSS preceding the peak centerline velocity by $0.05 t / \tau$. In Fig. 9(1) the offset is shown to increase in the compliant phantom to 0.07 $t / \tau$. The cause of this is due to the expansion and contraction of the phantom. It can be seen at peak expansion there is a severe drop in WSS magnitude. Referring back to Fig. 6 it can be seen that as the phantom dilates the velocity gradient in the proximity of the phantom wall reduces when compared to the rigid geometry. With WSS directly 
proportional to velocity gradient, the reduction in gradient reduces the WSS. Further it can be seen that the WSS reduces to the minimum value experienced in the cardiac cycle at peak centerline velocity (equivalent to peak flow rate), which is not observed in the rigid results. There are however varying results when looking at the effect compliance has on the minimum WSS as little variation was observed. It is expected at low Re that WSS predictions between compliant and rigid geometries would be similar as the internal diameter of the compliant geometry would be similar to the rigid. When comparing the compliant WSS results to that of previous in-vitro and in-vivo studies it can be seen the maximum, minimum and TAWSS of $1.4,0.58$ and $0.84 \mathrm{~Pa}$ in the compliant phantom are in good agreement with the in-vivo results obtained through MRI by Zhang et al. ${ }^{52}$. It should be noted that pressure data was not recorded with this investigation. Future work should incorporate pressure sensors in the experimental set up. This is important as pressure variation drives expansion and contraction. For an $\alpha$ of 4.54 a small phase lag is expected ${ }^{40}$ between pressure and flow rate. Observing pressure data, would help elucidate further the effects compliance has on WSS and add further validity to the matching of invivo conditions.

$$
F(t)_{n o r m}=\frac{2(F(t)-\bar{F}(t))}{\max (F(t)-\bar{F}(t))-\min (F(t)-\bar{F}(t))} .
$$
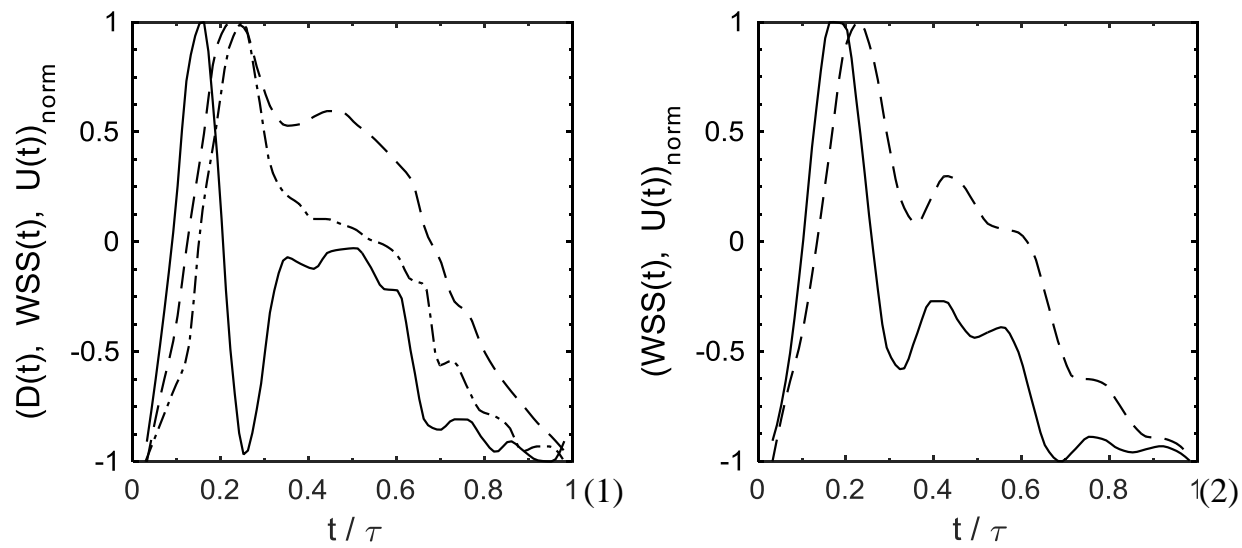

Fig. 9. (1) Temporal evolution of the normalized WSS (-), centerline velocity (--) and Diameter (--) in the compliant phantom. (2) Temporal evolution of the normalized WSS (-), centerline velocity (--) in the rigid phantom.

\section{Conclusion}

This study has provided a direct comparison of both the flow field and WSS observed in an experimental phantom of rigid and compliant geometry. The results of the present work have shown that a rigid boundary causes a severe over estimation of the peak WSS and TAWSS experienced. In a healthy geometry the introduction of compliance reduces peak WSS by $40 \%$. Compliance reduces the magnitude of WSS to its minimum value at peak flow rate which does not occur with a rigid assumption. There is also a $3 \%$ increase in phase lead of peak WSS to peak centerline velocity. This shows that compliance is integral to the understanding of arterial fluid dynamics, as it provides an amelioration, by reducing the magnitude of the oscillation of WSS which can exacerbate atherosclerosis ${ }^{29}$. However compliance has little or no effect on minimum WSS. Numerical codes reported 
in the literature have been used only to provide preliminary studies so far. There is an obvious need for good data to validate them.

\section{Acknowledgements}

We are grateful to Mr Julian Phillips and the staff of the Dept. Of Mechanical Engineering workshop for technical support. Author PHG was supported by a UC Doctoral scholarship.

\section{References}

[1] Mozaffarian, D, Benjamin, EJ, Go, AS, Arnett, DK, Blaha, MJ, Cushman, M, Das, SR, de Ferranti, S, Després, J-P and Fullerton, HJ, Heart Disease and Stroke Statistics-2016 Update A Report From the American Heart Association, Circulation:CIR. 0000000000000350, 2015

[2] Traub, O and Berk, BC, Laminar Shear Stress : Mechanisms by Which Endothelial Cells Transduce an Atheroprotective Force, Arterioscler Thromb Vasc Biol, 18(5):677-685, 1998

[3] Chatzizisis, YS, Coskun, AU, Jonas, M, Edelman, ER, Feldman, CL and Stone, PH, Role of Endothelial Shear Stress in the Natural History of Coronary Atherosclerosis and Vascular Remodeling: Molecular, Cellular, and Vascular Behavior, Journal of the American College of Cardiology, 49(25):2379-2393, 2007

[4] Ku, DN, Blood flow in arteries, Annual Review of Fluid Mechanics, 29:399-434, 1997

[5] Taylor, CA and Draney, MT, Experimental and Computational Methods in Cardiovascular Fluid Mechanics, Annual Review of Fluid Mechanics, 36(1):197231, 2004

[6] Yamaguchi, T, Ishikawa, T, Tsubota, K-i, Imai, Y, Nakamura, M and Fukui, T, Computational Blood Flow Analysis - New Trends and Methods, Journal of Biomechanical Science and Engineering, 1(1):29-50, 2006

[7] Taylor, CA and Figueroa, CA, Patient-Specific Modeling of Cardiovascular Mechanics, Annual Review of Biomedical Engineering, 11(1):109-134, 2009

[8] Vennemann, P, Lindken, R and Westerweel, J, In vivo whole-field blood velocity measurement techniques, Experiments in Fluids, 42(4):495-511, 2007

[9] Elkins, C and Alley, M, Magnetic resonance velocimetry: applications of magnetic resonance imaging in the measurement of fluid motion, Experiments in Fluids, 43(6):823-858, 2007

[10] Sherwin, SJ and Blackburn, HM, Three-dimensional instabilities and transition of steady and pulsatile axisymmetric stenotic flows, Journal of Fluid Mechanics, 533:297-327, 2005

[11] Varghese, SS, Frankel, SH and Fischer, PF, Direct numerical simulation of stenotic flows, Part 2 : Pulsatile flow, Journal of Fluid Mechanics, 582:281-318, 2007

[12] Hoi, Y, Wasserman, BA, Lakatta, EG and Steinman, DA, Effect of Common Carotid Artery Inlet Length on Normal Carotid Bifurcation Hemodynamics, Journal of Biomechanical Engineering, 132(12):121008-121008, 2010

[13] Mao, X, Sherwin, S and Blackburn, H, Transient growth and bypass transition in stenotic flow with a physiological waveform, Theoretical and Computational Fluid Dynamics, 25(1):31-42, 2011 
[14] Dhahbi, M, Ben Chiekh, M, Gilles, B, Béra, JC and Jemni, A, Numerical simulations of particle dynamics in a poststenotic blood vessel region within the scope of extracorporeal ultrasound stenosis treatment, Medical Engineering \& Physics, 34(7):982-989, 2012

[15] Xiong, G, Figueroa, CA, Xiao, N and Taylor, CA, Simulation of blood flow in deformable vessels using subject-specific geometry and spatially varying wall properties, International Journal for Numerical Methods in Biomedical Engineering, 27(7):1000-1016, 2011

[16] Moireau, P, Bertoglio, C, Xiao, N, Figueroa, C, Taylor, C, Chapelle, D and Gerbeau, JF, Sequential identification of boundary support parameters in a fluidstructure vascular model using patient image data, Biomechanics and Modeling in Mechanobiology:1-22, 2012

[17] Molla, MM and Paul, MC, LES of non-Newtonian physiological blood flow in a model of arterial stenosis, Medical Engineering \& Physics, 34(8):1079-1087, 2012

[18] Saeid Khalafvand, S and Han, H-C, Stability of Carotid Artery Under Steady-State and Pulsatile Blood Flow: A Fluid-Structure Interaction Study, Journal of Biomechanical Engineering, 137(6):061007-061007, 2015

[19] Kung, E, Les, A, Figueroa, C, Medina, F, Arcaute, K, Wicker, R, McConnell, M and Taylor, C, In Vitro Validation of Finite Element Analysis of Blood Flow in Deformable Models, Annals of Biomedical Engineering, 39(7):1947-1960, 2011

[20] Geoghegan, PH, Buchmann, NA, Soria, J and Jermy, MC, Time-resolved PIV measurements of the flow field in a stenosed, compliant arterial model, Experiments in Fluids, 54(5):1-19, 2013

[21] Pielhop, K, Klaas, M and Schröder, W, Experimental analysis of the fluid-structure interaction in finite-length straight elastic vessels, European Journal of Mechanics - B/Fluids, 50:71-88, 2015

[22] Perktold, K and Rappitsch, G, Computer simulation of local blood flow and vessel mechanics in a compliant carotid artery bifurcation model, Journal of Biomechanics, 28(7):845-856, 1995

[23] Gerbeau, J-F, Vidrascu, M and Frey, P, Fluid-structure interaction in blood flows on geometries based on medical imaging, Computers \& Structures, 83(23):155-165, 2005

[24] Tezduyar, TE, Sathe, S, Cragin, T, Nanna, B, Conklin, BS, Pausewang, J and Schwaab, M, Modelling of fluid-structure interactions with the space-time finite elements: Arterial fluid mechanics, International Journal for Numerical Methods in Fluids, 54(6-8):901-922, 2007

[25] Tezduyar, TE, Sathe, S, Schwaab, M and Conklin, BS, Arterial fluid mechanics modeling with the stabilized space-time fluid-structure interaction technique, International Journal for Numerical Methods in Fluids, 57(5):601-629, 2008

[26] Takizawa, K, Christopher, J, Tezduyar, TE and Sathe, S, Space-time finite element computation of arterial fluid-structure interactions with patient-specific data, International Journal for Numerical Methods in Biomedical Engineering, 26(1):101-116, 2010

[27] Bertram, CD and Elliott, NSJ, Flow-rate limitation in a uniform thin-walled collapsible tube, with comparison to a uniform thick-walled tube and a tube of tapering thickness, Journal of Fluids and Structures, 17(4):541-559, 2003

[28] Bertram, CD and Nugent, AH, The Flow Field Downstream of an Oscillating Collapsed Tube, Journal of Biomechanical Engineering, 127(1):39-45, 2005 
[29] Ku, DN, Giddens, DP, Zarins, CK and Glagov, S, Pulsatile flow and atherosclerosis in the human carotid bifurcation. Positive correlation between plaque location and low oscillating shear stress, Arteriosclerosis, Thrombosis, and Vascular Biology, 5(3):293-302, 1985

[30] Eguchi, T, Satoshi, W, Hisasada, T and Akinori, F, Development of Pulsatile Flow Experiment System and PIV Measurement in an Elastic Tube, Memoirs of the Faculty of Engineering, Kyushu University, 63(3), 2003

[31] Geoghegan, PH, Buchmann, N, Jermy, M, Nobes, D, Spence, C and Docherty, PD, SPIV and image correlation measurements of surface displacement during pulsatile flow in models of compliant, healthy and stenosed arteries Proc. 15th International Symposium of Laser Techniques to Fluid Mechanics. Lisbon, Portugal 2010,

[32] Burgmann, S, Große, S, Schröder, W, Roggenkamp, J, Jansen, S, Gräf, F and Büsen, M, A refractive index-matched facility for fluid-structure interaction studies of pulsatile and oscillating flow in elastic vessels of adjustable compliance, Experiments in Fluids, 47(4):865-881, 2009

[33] Yagi, T, Kamoda, A, Sato, A, Yang, W and Umezu, M, 3D volume flow visualization for vascular flow modelling using stereo PIV with fluorescent tracer particles Proc. 8th International Symposium on particle image Velocimetry (PIV 09). Melbourne, Australia 2009,

[34] Ding, Z, Wang, K, Li, J and Cong, X, Flow field and oscillatory shear stress in a tuning-fork-shaped model of the average human carotid bifurcation, Journal of Biomechanics, 34(12):1555-1562, 2001

[35] Long, Q, Xu, XY, Ramnarine, KV and Hoskins, P, Numerical investigation of physiologically realistic pulsatile flow through arterial stenosis, Journal of Biomechanics, 34(10):1229-1242, 2001

[36] Cao, P, Duhamel, Y, Olympe, G, Ramond, B and Langevin, F, A new production method of elastic silicone carotid phantom based on MRI acquisition using rapid prototyping technique Proc. Engineering in Medicine and Biology Society (EMBC), 2013 35th Annual International Conference of the IEEE. 2013,

[37] Geoghegan, PH, Buchmann, NA, Spence, CJT, Moore, S and Jermy, M, Fabrication of rigid and flexible refractive-index-matched flow phantoms for flow visualisation and optical flow measurements, Experiments in Fluids, 52(5):13311347, 2012

[38] Caro, CG, Pedley, TJ, Schroter, RC and Seed, WA 2012, The mechanics of the circulation: Second Edition. Cambridge University Press.

[39] Riley, W, Barnes, R, Evans, G and Burke, G, Ultrasonic measurement of the elastic modulus of the common carotid artery. The Atherosclerosis Risk in Communities (ARIC) Study, Stroke, 23(7):952-956, 1992

[40] Womersley, JR, Method for the calculation of velocity, rate of flow and viscous drag in arteries when the pressure gradient is known, Journal of Physiology, (127):553-563, 1955

[41] Buchmann, NA and Jermy, MC, Transient Flow and Shear Stress Measurements in an anatomical Model of the Human Carotid Artery Proc. 15th International Symposium of Laser Techniques to Fluid Mechanics. Lisbon, Portugal 2010,

[42] Guyton, AC and Hall, JE 2000, Textbook of Medical Physiology. W. B. Saunders Company.

[43] Fung, YC 1993, Biomechanics: Mechanical Properties of Living Tissues, 2nd ed. 175 Fifth Avenue, New York, NY 10010, USA: Springer-Verlag New York, Inc. 
[44] Spence, CJT, Buchmann, NA and Jermy, MC, Unsteady flow in the nasal cavity with high flow therapy measured by stereoscopic PIV, Experiments in Fluids, 52(3):569-579, 2012

[45] Raffel, M, Willert, CE, Wereley, ST and Kompenhans, J 2007, Particle image velocimetry: a practical guide second edition. 2nd ed.: Springer (Berlin and New York). xvi, 253.

[46] Buchmann, NA and Jermy, MC, Particle image velocimetry measurements of blood flow in a modeled carotid artery bifurcation Proc. 16th Australasian Fluid Mechanics Conference. Gold Coast, Australia 2007,

[47] Westerweel, J and Scarano, F, Universal outlier detection for PIV data, Experiments in Fluids, 39(6):1096-1100, 2005

[48] Buchmann, NA, Jermy, MC and Nguyen, CV, Experimental investigation of carotid artery haemodynamics in an anatomically realistic model, Int. J. Exp. Comp. Methods in Biomechanics 1(2):172-192, 2009

[49] Buchmann, NA, Yamamoto, M, Jermy, M and David, T, Particle Image Velocimetry (PIV) and Computational Fluid Dynamics (CFD) Modelling of Carotid Artery Haemodynamics under Steady Flow: A Validation Study, Journal of Biomechanical Science and Engineering, 5(4):421-436, 2010

[50] Sui, B, Gao, P, Lin, Y, Qin, H, Liu, L and Liu, G, Noninvasive determination of spatial distribution and temporal gradient of wall shear stress at common carotid artery, Journal of Biomechanics, 41(14):3024-3030, 2008

[51] Cao, J and Rittgers, S, Particle Motion Within In Vitro Models of Stenosed Internal Carotid and Left Anterior Descending Coronary Arteries, Annals of Biomedical Engineering, 26(2):190-199, 1998

[52] Zhang, F, Barker, AJ, Gates, PE, Strain, WD, Fulford, J, Mazzaro, L, Shore, AC, Bellenger, NG, Lanning, $\mathrm{C}$ and Shandas, R, Noninvasive wall shear stress measurements in human carotid artery using echo particle image velocimetry: Initial clinical studies Proc. Ultrasonics Symposium (IUS), IEEE International. 2009, 